\title{
Analysis and Segmentation of Remote-Sensing Images for Land-Cover Mapping
}

\author{
P.C. Smits and S.B. Serpico \\ University of Genoa \\ Department of Biophysical and Electronic Engineering \\ Via all'Opera Pia 11A, 16145 Genova, Italy \\ \{smits,vulcano\}@dibe.unige.it
}

\begin{abstract}
In the framework of the European Community programme Training and Mobility for Researchers, the project Analysis and Segmentation of Remote-Sensing Images for Land-Cover mapping has been proposed and approved. This article provides some insight in the role of pattern recognition and image processing techniques in the European remote-sensing community and gives and overview of the project's objectives and results to date.
\end{abstract}

\section{Remote-sensing and image analysis}

Remote Sensing exists by the virtue of physical, economical, political or other constraints that prevent human beings from exploring certain regions. Although many sophisticated instruments have been developed, the hunger for information seems more than ever unsatisfyable, and the more types of information can be extracted from new or existing remote-sensing images, the more economical attractive these data become.

It is not our intention to go too much into detail on all the aspects that are related to remote-sensing image analysis. We will limit ourselves to some observations that will help to understand the scientific direction of the project Analysis and Segmentation of Remote-Sensing Images for Land-Cover mapping (ANRS). For an up-to-date, complete and comprehensive textbook on remote-sensing image analysis the reader is referred to [1].

Segmentation, classification, and change-detection of remote-sensing images can be formulated as ill-posed problems. Although the quality of many modern sensors is such that these problems are not too ill-posed, this situation changes in cases where one wants to extract types of information for which the sensor has not been build in the first place. This can be the case, for example, when one is interested in the recognition of objects with dimensions close to the pixel resolution.

When dealing with complex natural and agricultural landscapes, characterized by the presence of mixed classes and by land-cover/ground responses influenced by terrain topographies, it is not sufficient to base decisions regarding a data element only on the single element itself coming from a single sensor. Regularization of the ill-posed analysis problem is needed such that the uncertainty is reduced sharply, thus improving the consistency of the solution. The information needed to regularize a problem can come, for instance, from 
application specific a-priori knowledge about the imaged objects (for instance modelled into a so-called smoothness constraints) or from other types of data.

In the ANRS project, special attention is given to the family of Markov Random Field (MRF) approaches, that allow one to combine statistical image models and regularization constraints for a huge range of image analysis problems.

Multisensorial approaches are being investigated for a synergetic exploitation of different types of information provided by different input channels (e.g., the morphological, topological and spatial information present in different polarizations of radar sensors, different observation frequencies, optical sensors). To further improve classification accuracy, classification approaches that can explicitly take into account the class mixture will also be considered. Their usefulness is evident in applications where classes are not well separated and their number is very large. This innovative approach to classification is known in the literature as "mixed classification".

Table I gives an overview of conventional civil satellite sensors and their utilization. In the ANSR project, synthetic aperture radar (SAR) data plays a significant role because of its growing exploitation.

Table I. Data sources that will be considered in the ANSR project.

\begin{tabular}{|l|l|l|}
\hline Data & Technical Requirements & Utilisation \\
\hline Landsat TM & $\begin{array}{l}\text { Swath } 185 \mathrm{~km}, \text { resolution } \\
30 \mathrm{~m} .\end{array}$ & $\begin{array}{l}\text { Merging with high spatial resolution } \\
\text { data for land-cover/ use; fusion with } \\
\text { optical data }\end{array}$ \\
\hline ERS1/2 SAR & $\begin{array}{l}\text { Swath } 100 \mathrm{~km}, \text { resolution } \\
25 \mathrm{~m} . \\
\text { C-band, VV }\end{array}$ & $\begin{array}{l}\text { Occurrence in flash floods and } \\
\text { change detection }\end{array}$ \\
\hline SPOT 2 & High resolution & Land mapping and change detection \\
Scansar & $\begin{array}{l}\text { Swath width } 500 \mathrm{~km}, \text { spatial } \\
\text { resolution } 100 \mathrm{~m} \text { for } \\
\text { microwave imaging }\end{array}$ & $\begin{array}{l}\text { Fusion with AVHRR/NOAA or } \\
\text { with Landsat for assessment of soil } \\
\text { types, and catchment areas }\end{array}$ \\
\hline Radarsat & $\begin{array}{l}\text { Swath } 100 \mathrm{~km}, \text { resolution } \\
25 \mathrm{~m} \\
\text { C-band HH }\end{array}$ & $\begin{array}{l}\text { Fusion with ERS1/2 and optical } \\
\text { data for extraction of vegetation and } \\
\text { soil properties }\end{array}$ \\
\hline JERS-1 SAR & $\begin{array}{l}\text { Swath } 185 \mathrm{~km}, \text { resolution } \\
18 \mathrm{~m} \\
\text { L-band } \mathrm{HH}\end{array}$ & $\begin{array}{l}\text { Fusion with ERS1/2 and optical } \\
\text { data for extraction of vegetation and } \\
\text { soil properties }\end{array}$ \\
\hline
\end{tabular}

Now that policy makers focus their attention more and more on environmental issues, the technology for monitoring the environmental resources has gained importance. In this demanding and ever-changing field the necessity of a systematic and goal directed development of imaging technology is felt strongly. Below follow some citations from EC programmes that illustrate this.

One objective of the Environment and Climate programme is ([2], p. 5) the contribution to the technological development necessary for environmental observation, monitoring and research, including methodologies and technologies 
for the monitoring, warning and management of natural hazards. The objectives of Area 3.1 in theme 3 (Methodological research and pilot projects) are to improve and properly target European technical and scientific capability in Earth Observation (EO) data treatment and interpretation; to develop applications of $E O$ data of European interest, and to improve the quality and cost-effectiveness of the derived information ([2], pp. 47-48). In section 3.1.1 (Methodological research), attention is given to the development of generic tools and techniques for automated pre-processing, interpretation and integration of remotely sensed and ground data, covering techniques for image segmentation and feature extraction and classification (including the systematic use of auxiliary data and contextual information).

Links can be found with the "Agriculture and Fisheries programme" as well. Besides the exchange of scientific and technological know-how, area 4.2 (quality policy) focuses on the Technological instruments and methods (area 4.2.2). Furthermore, the activities of the project are related with those of the Joint Research Centre - Institute for Remote Sensing Applications (IRSA).

\section{ANSR's objectives}

The objective of the ANSR is to develop robust and sufficiently accurate methods for the segmentation, classification and change detection of remotely sensed data for land-cover mapping. The following sub-objectives have been defined:

- To define a methodology to assess the quality of segmentation, classification and change-detection methods for land-cover applications.

- To develop and evaluate segmentation, classification and change detection methods that should be more cost-effective than existing methods.

Some of the innovative aspects of the projects are:

- The explicit definition of quality measures regarding the segmentation and classification of land-cover remote-sensing data, in co-operation with users in the field;

- The extensive use of different types of information present in the data;

- High-levels of user-friendliness and robustness provided by a high degree of automation of the estimation of processing parameters that will aid a future implementation in geographical information systems (GISs);

- The project aims at the production of more detailed thematic maps, through the refinement of processing techniques rather than the utilization of highperformance (and expensive) acquisition instruments. 


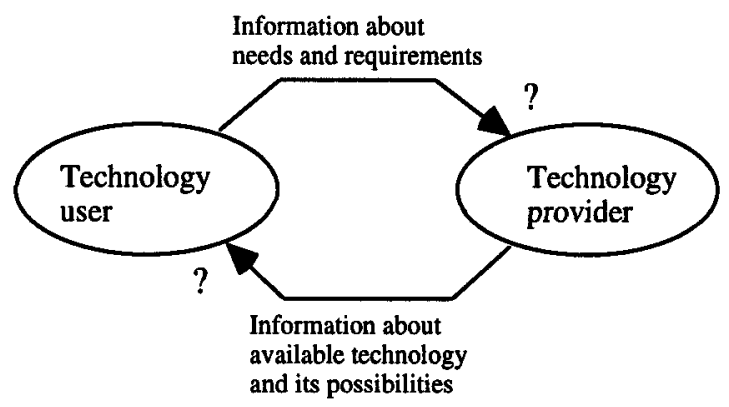

Fig. 1. Mutual understanding between technology users and providers is needed for an efficient, goal-directed development of technology.

\section{Results to date}

The ANSR project has been split into three parts or Work Packages: 1) quality assessment; 2) development of suited methods; and 3) evaluation, validation and dissemination of the results.

An important part of the research regarding the quality assessment of remotesensing image classification has been done in collaboration with the Digital Image Analysis Lab, University of Arizona, Tucson (AZ). This study consisted of an extended reviewed of accuracy assessment methods for image classification algorithms. The concept of accuracy has been extrapolated to that of quality, being aware that we cannot do this except by creating the link between the accuracy and the user objectives and requirements (Fig. 2). Therefore, the following issues have been addressed: who the end-user really is, what his requirements are, and to what extend he is aware of the costs involved and how these costs can be fed back to the objectives.

It became clear that in remote-sensing it is very difficult to objectively validate image classification result because the semantics of the class labels in training and test data often do not adequately represent the actual physical reality. Moreover, these data are very expensive. Therefore a relative validation in which classifiers are compared is found more appropriate than an absolute one. Furthermore, the study revealed that most end-users who apply remote-sensing imagery to create thematic maps require a class-accuracy of $90 \%$ or better (many even $95 \%$ or better). Accuracy is found very important, as it is the last thing that people would make compromises on. 


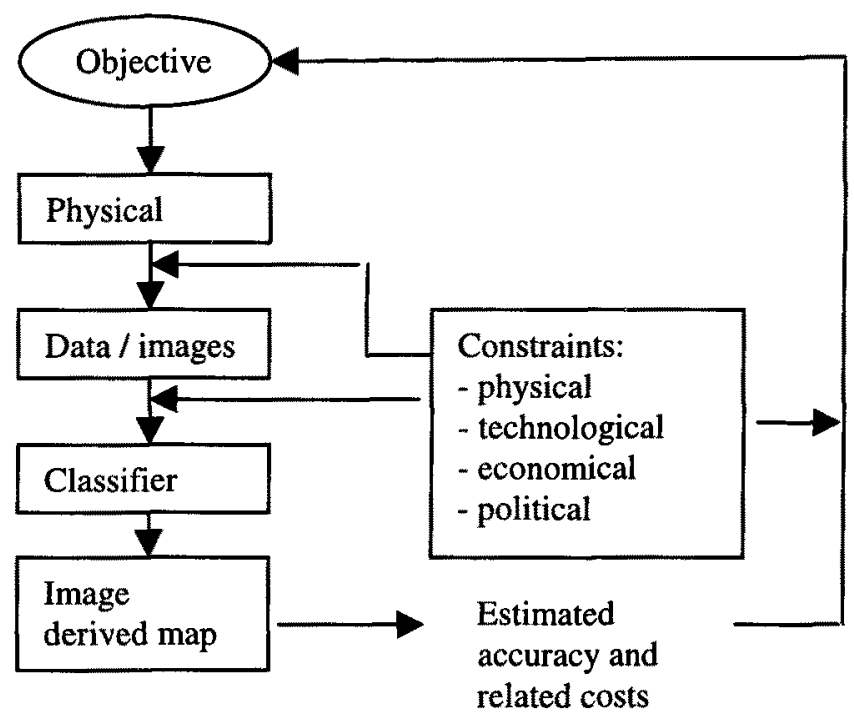

Fig. 2. Interrelationship between the objective and different aspects of remote-sensing derived maps.

Work Package 2 focuses on the development of image analysis methodologies. The MRF approach has proven to be very successful in addressing the issues of both data modelling and regularization in segmentation and classification by means of a-priori information and other sources of data (e.g., digital read maps, optical data, etc.)[2],[3],[4],[5],[6]. But also filtering can be performed by using suited MRF models, as has been shown in [9] and [12]. Recent research aims at reducing the computational burden that normally comes with iteratively minimizing the MRF models, and exploits causal Markov mesh models that have real-time capabilities [11].

\section{Acknowledgements}

This work was supported by the European Community programme Training and Mobility for Researchers (Marie Curie Fellowship) under Contract ERBFM BICT 950257. Part of the project was performed during two visits by the first author to the Digital Image Analysis Lab, University of Arizona, Tucson (AZ).

More information on the TMR programme and the Marie Curie Fellowship can be found at URL http: / / www2 . cordis . lu/tmr/home.html

Up-to-date information on the ANSR project can be found at URL http://dibe.unige.it/TMR_Smits 


\section{References}

[1] R. A. Schowengerdt (1997). Remote Sensing - Models and methods for image processing. Academic Press, 1997

[2] Environment and Climate 1994-1998 Work programme, European Commission, 1994.

[3] P.C. Smits and S.G. Dellepiane, "Synthetic Aperture Radar image segmentation by a detail preserving Markov Random Field appraoch," IEEE Transactions on Geoscience and Remote Sensing (in press, scheduled for July 1997).

[4] P.C. Smits and S. Dellepiane, "Markov Random Field based image segmentation with adaptive neighborhoods to the detection of fine structures in SAR data," Int. Geoscience and Remote Sensing Symposium, Lincoln, May 1996, pp. 714-716.

[5] P.C. Smits and S. Dellepiane, "Information fusion in a Markov Random Field based image segmentation approach using adaptive neighbourhoods," Int. Conf. on Pattern Recognition, Vienna, August 1996, pp. 570-575.

[6] P.C. Smits and S. Dellepiane, "Data fusion in a Markov Random Field based image segmentation approach," European Symposium on Satellite Remote Sensing, Toarmina, Italy, 23-27 Sept., 1996, pp. 85-95.

[7] P.C. Smits, A. Trucco, G. Bo, G. Vernazza, "Iterative model reconstruction for phase unwrapping," Proc. of the third ERS Scientific Symposium, Florence, Italy, 17-20 March 1997 (in press).

[8] P.C. Smits and S. Dellepiane, "An irregular MRF region label model for multi-channel image segmentation," Proc. of the Conference Pattern Recognition in Practice V, Vlieland, Holland, 4-6 June, 1997 (in press).

[9] P.C. Smits and S. Dellepiane, "Discontinuity adaptive MRF model for remote-sensing image analysis," Proc. of the 1997 Int. Geoscience and Remote Sensing Symposium (IGARSS'97), Singapore, Aug. 1997 (in press).

[10] P.C. Smits, R. Vaccaro, and S. Dellepiane, "Automated hierarchical classification of SAR images," Proc. of the 1997 Int. Geoscience and Remote Sensing Symposium (IGARSS97), Singapore, Aug. 1997 (in press).

[11] P.C. Smits, A. Martuccelli, F. Giorgini, M. Petrou, "SAR image interpretation based on hidden Markov mesh random field models," Proc. of the 1997 Int. Geoscience and Remote Sensing Symposium (IGARSS'97), Singapore, Aug. 1997 (in press).

[12] P.C. Smits, S.G. Dellepiane, and G. Vernazza, "Discontinuity adaptive MRF model for Synthetic Aperture Radar image analysis," Proc. $9^{\text {th }}$ International Conference on Image Analysis and Processing, 17-19 September 1997, Florence, Italy (accepted for publication). 